\title{
SGK protein kinase facilitates the expression of long-term potentiation in hippocampal neurons
}

\author{
Yun L. Ma, Ming C. Tsai, Wei L. Hsu, and Eminy H.Y. Lee ${ }^{1}$ \\ Division of Neuroscience, Institute of Biomedical Sciences, Academia Sinica, Taipei, Taiwan, Republic of China
}

\begin{abstract}
Previous studies showed that the serum- and glucocorticoid-inducible kinase ( $\mathrm{ggk}$ ) gene plays an important role in long-term memory formation. The present study further examined the role of SGK in long-term potentiation (LTP). The dominant-negative mutant of sgk, SGKS422A, was used to inactivate SGK. Results revealed a time-dependent increase in SGK phosphorylation after tetanization with a significant effect observed $3 \mathrm{~h}$ and $5 \mathrm{~h}$ later. Transfection of SGKS422A impaired the expression, but not the induction, of LTP. Furthermore, the constitutively active sgk, SGKS422D, up-regulated postsynaptic density-95 expression in the hippocampus. These results together support the role of SGK in neuronal plasticity.
\end{abstract}

The serum- and glucocorticoid-inducible kinase $(s g k)$ gene is a member of the serine/threonine protein kinase gene family that is transcriptionally induced by glucocorticoid and serum (Webster et al. 1993). The sgk gene was well known for its role in regulation of sodium transport (Naray-Fejes-Toth et al. 2000; Pearce et al. 2000) and cell volume (Waldegger et al. 1997) in renal epithelia. But its role in the central nervous system is less known. In our previous study, we have shown that the sgk gene plays an important role in memory formation of spatial learning in rats. The expression level of this gene is significantly higher in the dorsal hippocampus of fast learners than slow learners from the water maze learning task (Tsai et al. 2002). Furthermore, transient transfection of the dominant-negative mutant of $s g k$, SGKS422A, to hippocampal CA1 neurons markedly impaired, whereas transfection of the $s g k$ wild-type DNA facilitated spatial memory formation in rats (Tsai et al. 2002).

More recently, we have found that environmental enrichment training significantly enhanced $s g k$ expression in the hippocampus. Enrichment training also reversed SGKS422A mutant DNA-induced impairment in spatial learning, fear-conditioning learning, and novel object-recognition learning in rats (Lee et al. 2003). These results together suggest that SGK may play an important role in neuronal plasticity. In the present study, we further examined this issue by studying the role of SGK in long-term potentiation (LTP), a synaptic model for long-term memory formation (Bliss and Collingridge 1993), in hippocampal neurons.

The dominant-negative form of SGK (SGKS422A) and the constitutively active form of SGK (SGKS422D) (Kobayashi and Cohen 1999) were used as molecular tools to inactivate and activate SGK at Ser422, respectively. SGKS422A was transfected to the rat CA1 area, and these animals were sacrificed at different time points after DNA transfection. SGK phosphorylation at Ser422 was evaluated by Western blot using the specific phospho (p)422SGK antibody. Results revealed that SGKS422A transfection produced a significant decrease in SGK Ser422 phosphorylation $48 \mathrm{~h}$ and $72 \mathrm{~h}$ later $\left(F_{5,12}=38.22, P<0.01, \mathrm{tD}=4.56\right.$ and 9.5, respectively, $P<0.05$ and $P<0.01$ ) (Fig. 1A,B). This effect was recovered $96 \mathrm{~h}$ after DNA transfection $(\mathrm{tD}=1.81, P>0.05)$ (Fig. 1A,B). These results indicated that SGKS422A transfection produced a transient and time-dependent decrease in SGK phosphorylation at Ser422. Since SGK shares high homology in its

'Corresponding author.

E-mail eminy@gate.sinica.edu.tw; fax 886-2-2782-9224.

Article and publication are at http://www.learnmem.org/cgi/doi/10.1101/ Im.179206. kinase domain to that of Akt (Brazil and Hemmings 2001), and phosphoinositide-dependent kinase 1 (PDK1)/PDK2 directly phosphorylates SGK at Thr256/Ser422 and Akt at Thr308/Ser473 (Kobayashi and Cohen 1999), we wondered whether SGKS422A transfection may also inhibit Akt phosphorylation due to mechanisms such as competition for PDK1/PDK2. We next examined this possibility. Results revealed that at the 48-h and 72-h time points at which SGKS422A transfection markedly decreased Ser422 SGK phosphorylation, it did not affect Ser473 Akt phosphorylation (Fig. 1C,D). These results suggest that the effect of SGK mutant DNA transfection was specific for SGK and did not also block Akt signaling.

To confirm that SGKS422A was transfected and expressed in CA1 neurons, immunohistochemistry was carried out by using antibody against the HA-tagged protein and FITC-conjugated IgG secondary antibody. Results revealed that when HASGKS422A mutant DNA was transfected to the CA1 area, specific fluorescence labeling was observed (Fig. 1E).

Because LTP serves as a synaptic model for long-term memory formation, in the present study, we examined the relationship between LTP and SGK. Animals were divided into five groups ( $n=4-6$ each group) and subjected to tetanic stimulation. Electrophysiological recording lasted for different time periods (control, $10 \mathrm{~min}, 1 \mathrm{~h}, 3 \mathrm{~h}$, and $5 \mathrm{~h}$, respectively) (Fig. 2A). Animals were sacrificed immediately after recording, and the CA1 tissue containing the recording site was dissected out using a punch with $2 \mathrm{~mm}$ diameter for Western blot analysis of the p422 SGK level. Results revealed that there was a time-dependent change in Ser422 SGK phosphorylation after LTP induction $\left(F_{4,22}=2.93, P<0.05\right)$ (Fig. 2 B). Further analyses indicated that SGK phosphorylation at Ser422 was significantly increased at $3 \mathrm{~h}$ and $5 \mathrm{~h}(\mathrm{tD}=2.74$ and 2.92, respectively, both $P<0.05)$ (Fig. $2 \mathrm{~B})$, but not at $10 \mathrm{~min}$ and $1 \mathrm{~h}$ after LTP induction (both $P>0.05$ ) (Fig. 2B).

The above results demonstrated a time-dependent increase in Ser422 SGK phosphorylation correlated with LTP expression. But these results do not show a causal relationship between these two. In the next experiment, pcDNA3 vector or SGKS422A mutant DNA was transfected to the CA1 area $48 \mathrm{~h}$ before tetanic stimulation followed by electrophysiological recording for $3 \mathrm{~h}$. Results revealed that SGKS422A transfection markedly impaired the expression of LTP $\left(F_{1,8}=14.8, P<0.01,120-180\right.$ min after tetanic stimulation) (Fig. $2 \mathrm{C}$ ) without significantly altering the induction of LTP $\left(F_{1,8}=0.30, P>0.05,0-10 \mathrm{~min}\right.$ after tetanic stimulation) (Fig. 2C). 
A

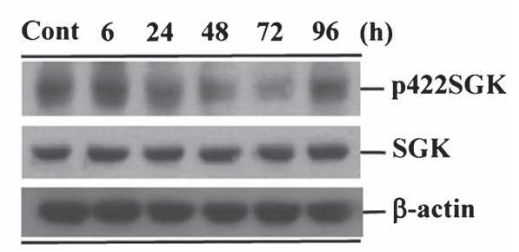

B

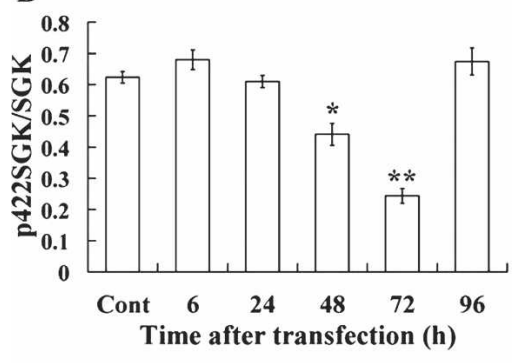

C

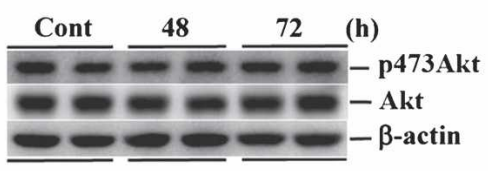

D

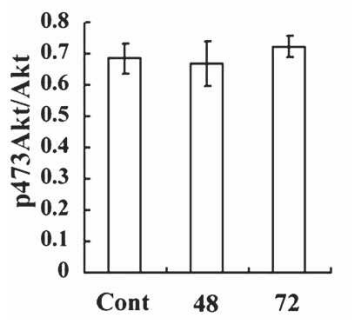

Time after transfection (h)

$\mathbf{E}$

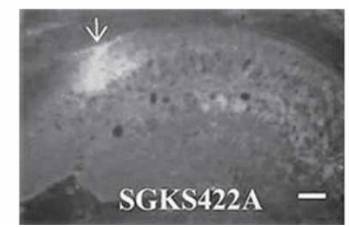

Figure 1. Time-course study of SGK phosphorylation after SGKS422A transfection. (A) Representative gel pattern showing p422SGK, SGK, and $\beta$-actin protein bands at different time points after SGKS422A transfection to the hippocampal CA1 area. (B) Statistical analysis revealed that SGKS422A transfection markedly decreased Ser422 SGK phosphorylation $48 \mathrm{~h}$ and $72 \mathrm{~h}$ later, but this effect is recovered at $96 \mathrm{~h}$. ${ }^{*} P<0.05$; ${ }^{* *} P<0.01$ (one-way ANOVA followed by Dunnett's $t$-test). (C) Representative gel pattern showing p473Akt, Akt, and $\beta$-actin protein bands $48 \mathrm{~h}$ and $72 \mathrm{~h}$ after SGKS422A transfection to CA1 area. (D) The same SGKS422A transfection did not alter Ser473 Akt phosphorylation at 48-h and 72-h time intervals. Data are mean \pm SEM. (E) Immunohistochemical staining showing fluorescence labeling in CA1 neurons upon HASGKS422A transfection to this area. An anti-HA primary antibody and a FITC-conjugated IgG secondary antibody were used. The arrow indicates the area of transfection. Bar, $220 \mu \mathrm{m}$.

Next, we examined whether SGK could regulate the expression of postsynaptic density-95 (PSD-95), a scaffolding protein that is enriched in the postsynaptic structure and is up-regulated upon synaptic activation (Okabe et al. 1999). Results revealed an overall significant effect of SGK on PSD-95 expression $\left(F_{2,12}=6.82, P<0.01\right)$ (Fig. 3). Further analyses indicated that transfection of SGKS422D significantly increased PSD-95 protein level in the hippocampus when compared with the pcDNA3 plasmid group $(P<0.01)$ (Fig. 3B). But transfection of pcDNA3 plasmid alone did not affect PSD-95 level $(P>0.05$ when compared with the PEI group) (Fig. 3B).

In the present study, we have used the dominant-negative mutant of the sgk gene, SGKS422A, as a molecular tool to study the role of SGK in hippocampal LTP. Results revealed that SGKS422A transfection to hippocampal CA1 neurons produced a transient and time-dependent decrease in SGK phosphorylation at Ser422, as indicated by using the specific phospho-422SGK antibody. This effect is evident at $48 \mathrm{~h}$ and $72 \mathrm{~h}$ after DNA transfection, which is consistent with an earlier report showing that transient DNA transfection has an optimal efficiency between 48 $\mathrm{h}$ and $72 \mathrm{~h}$ after injection (Abadall et al. 1996), and returns to the control level $96 \mathrm{~h}$ later. This time course was then adopted for manipulation of SGKS422A transfection and electrophysiological recording in the following experiment.

In examination of the role of $s g k$ involved in LTP, we found that SGK phosphorylation at Ser422 was significantly increased at a late stage of LTP recording, suggesting that $s g k$ probably plays a more important role in the expression of LTP. This speculation is supported by further experimental results showing that SGKS422A transfection impaired the expression of LTP without markedly affecting the induction of LTP (Fig. 2C). Because SGK is a downstream target of phosphatidylinositol 3-kinase (PI-3 kinase) and PDK1 signaling (Park et al. 1999), the present results are consistent with the report that PI-3 kinase is activated in response to tetanic stimulation and PI-3 kinase inhibitors block tetanus-induced LTP (Lin et al. 2001). On the other hand, although there seems a transient increase in SGK phosphorylation 30 min after LTP induction, a significant increase in SGK phosphorylation was not observed until $3 \mathrm{~h}$ later. These results are not consistent with the findings that Akt activation occurred faster upon LTP induction, and LTP was blocked earlier when PI-3 kinase inhibitor was given (Sanna et al. 2002). It is possible that a mechanism in addition to PI-3 kinase signaling is involved. For example, MAPK is activated upon LTP induction (English and Sweatt 1997), and MAPK was also shown to transcriptionally regulate sgk expression (Mizuno and Nishida 2001). Thus, the late-phase increase in SGK phosphorylation could possibly be due to an increase in $s g k$ gene expression.

In the present study, we have also found that transfection of SGKS422D up-regulated PSD-95 expression in the hippocampus. A possible explanation for this is translational regulation of PSD95 by SGK. In other studies, PSD-95 synthesis is similarly shown to be stimulated by estrogen (Akama and McEwen 2003) and insulin (Lee et al. 2005) via Akt signaling through translational regulation. PSD-95 is a scaffolding protein that is up-regulated upon synaptic activation (Okabe et al. 1999). Thus, our results also fit in well with a recent report showing that overexpression of constitutively active SGK increased dendritic growth of spinal cord neurons (David et al. 2005). Furthermore, our results are consistent with the notion that the late phase of LTP is associated with rearrangement of synaptic structures over time (Buchs and Muller 1996; Geinisman et al. 1996; Bolshakov et al. 1997). Moreover, PSD-95 was shown to control glutamate AMPA receptor incorporation during LTP (Ehrlich and Malinow 2004) and to increase AMPA receptor-mediated synaptic transmission in the cortex (Beique and Andrade 2003). We have recently found that AMPA receptor activation enhances $s g k$ mRNA expression in the hippocampus (Lee et al. 2003). In addition, AMPA receptormediated synaptic transmission is closely associated with the late phase of LTP (Nayak et al. 1998). These results together 
A

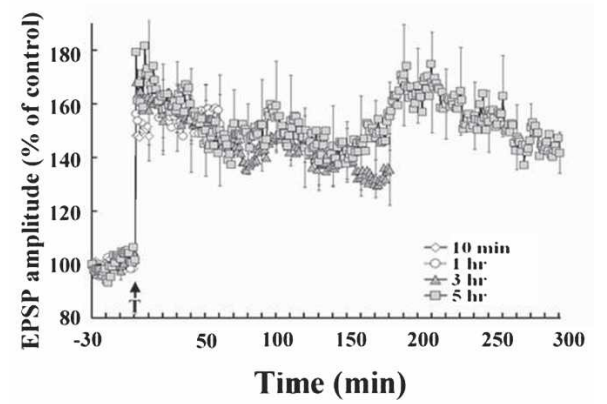

B

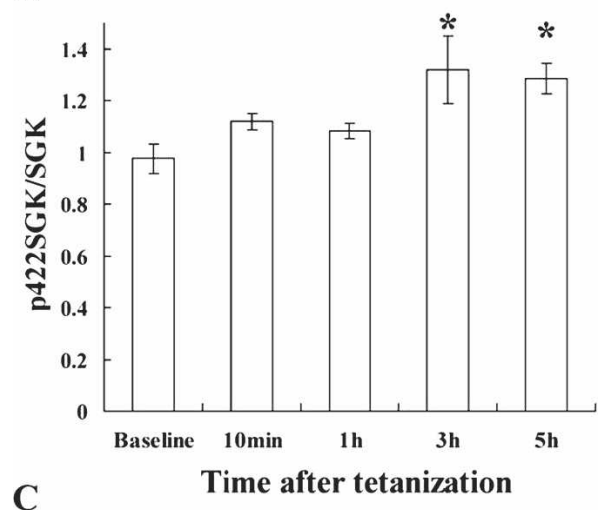

C

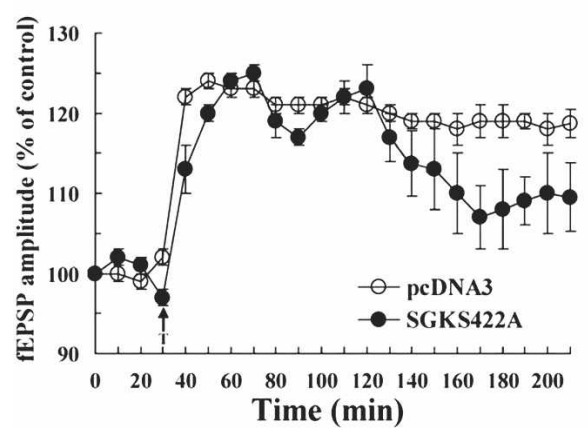

Figure 2. LTP induction increases SGK phosphorylation, and SGKS422A transfection impairs the expression of LTP. ( $A$ ) High-frequency stimulation of the Schaffer collateral/commissural pathway induces LTP at different time points. (T) Train of stimulation. (B) Western blot showed that SGK phosphorylation at Ser422 was significantly increased $3 \mathrm{~h}$ and 5 $\mathrm{h}$ after LTP induction (both $P<0.05$, one-way ANOVA followed by Dunnett's $t$-test). $N=4-6$ each group. (C) SGKS422A transfection $48 \mathrm{~h}$ before tetanic stimulation markedly impaired the expression, but not the induction, of LTP in hippocampal neurons $(P<0.01$, Student's $t$-test). $N=5$ each group. Data are mean \pm SEM.

further support the relationship between SGK and PSD-95 involved in synaptic plasticity. Moreover, because $s g k$ expression is mainly regulated by glucocorticoids (Webster et al. 1993), our result that SGK facilitated the expression of LTP not only supports our earlier finding that SGK facilitates long-term memory formation (Tsai et al. 2002), but also is consistent with the literature that glucocorticoids enhance memory consolidation in a variety of learning tasks in animals (for review, see Roozendaal 2000).

In the present study, the pcDNA3-transfected animals did not show the same magnitude of increase in LTP as that of the tetanization group (20\% vs. $45 \%$ increase). Both the plasmid DNA and polyethylenimine (PEI) are charged (negatively charged and positively charged, respectively). It is possible that the CA1 neurons are sensitive to the DNA/PEI charged complex that may affect the membrane excitability of these neurons. But whether the DNA/PEI complex actually affects CA1 neuron excitability and how it may do so requires further examination.

By using the current transfection method, the CA1 neurons affected are $-20 \%-25 \%$ viewed from a single plane (quantified by using the NIH IMAGE Program) and $<20 \%$ of total CA1 neurons in the dorsal hippocampus, but a significant blockade in the maintenance of LTP was observed. This is probably because the field potential was recorded only from several neurons in the CA1 area; if these neurons were located in the transfected area, significant electrophysiological effects would be observed. However, the significant decrease in SGK phosphorylation would need further explanation because the hippocampal tissue dissected out definitely contains many more neurons than have been recorded. The explanation for this discrepancy is not known yet, but several possibilities are considered. These include preferential transfection of DNA to certain specialized types of neurons in the hippocampus, alteration in hippocampal circuit properties, or an underestimation of transfected neurons. The exact mechanism requires further investigation.

In conclusion, we have demonstrated in the present study that transient transfection of the dominant-negative mutant of $s g k$, SGKS422A, to hippocampal CA1 neurons impaired the expression, but not the induction, of LTP. Furthermore, SGK activation up-regulated PSD-95 expression in the hippocampus. Several protein kinases have been shown to play a role in hippocampal LTP, such as MAPK (English and Sweatt 1997), cyclic AMP-dependent protein kinase (Nguyen and Woo 2003), calcium/calmodulin-dependent protein kinase II (Bejar et al. 2002), and PI-3 kinase (Opazo et al. 2003). The present study is the first report showing that protein kinase SGK also plays an important role in activity-dependent synaptic plasticity in hippocampal neurons.

Adult male Sprague-Dawley rats (250-300 g) bred in the Institute of Biomedical Sciences, Academia Sinica, Taiwan, were used. Animals were housed in a room maintained on a 12-h light/12-h dark cycle (light on at 6:30 a.m.) with food and water continuously available. Experimental procedures follow the Guidelines of Animal Use and Care of the National Institutes of Health.

For construction of the hemagglutinin (HA) epitope-tagged DNA plasmid (HA-SGK), full-length $s g k$ was cloned by amplifying the rat hippocampal sgk cDNA with primers 5'-CGGAATTC ACCGTCAAAACCGAGGCTCG-3' and 5'-GCTCTAGATCAGAGG AAGGAGTCCATAGG-3'. The PCR product was subcloned between the EcoRI and XbaI sites of the mammalian expression vector pcDNA3-HA. The kinase-deficient sgk mutant, SGKS422A, was generated by PCR with primers 5'-CGGAATTCACCGT CAAAACCGAGGCTCG-3' and 5'-GCTCTAGATCAGAGGAAG GAGTCCATAGGAGGGGCATAGGCGAAG-3', with HA-SGK as template and inserted into the pcDNA3-HA expression vector. The efficiency of $s g k$ mutant DNA transfection was confirmed by a decreased SGK activity in HEK293 cells (Park et al. 1999). The effect of SGKS422A DNA transfection was evaluated by SGK phosphorylation at Ser422 by using the phospho (p)-422SGK antibody. Site-directed mutagenesis was also carried out to generate the constitutively active form of $s g k$, SGKS422D. Before injection, plasmid DNA was diluted in 5\% glucose to a stock concentration of $5 \mu \mathrm{g} / \mu \mathrm{L}$. Branched polyethylenimine of $25 \mathrm{kDa}$ (Aldrich) was diluted to $0.1 \mathrm{M}$ in $5 \%$ glucose and added to the DNA solution. The final concentration of DNA injected was $1.5 \mu \mathrm{g} / \mu \mathrm{L}$ for $0.8 \mu \mathrm{L}$.

Animals were anesthetized with pentobarbital $(40 \mathrm{mg} / \mathrm{kg}$, i.p.) and subjected to stereotaxic surgery. Glucose solution (5\%) containing $1.2 \mu \mathrm{g}$ of plasmid DNA complexes with 10 PEI equivalents (Abadall et al. 1996) was injected to one side of the CA1 area 
A

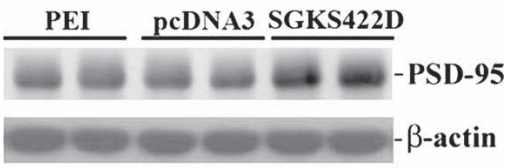

B

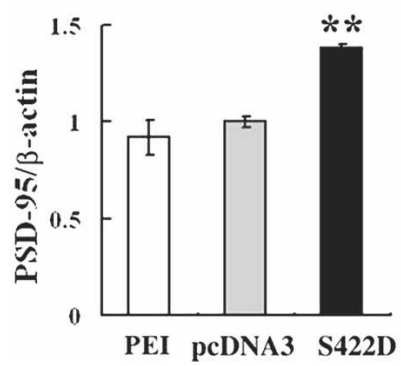

Figure 3. SGK up-regulates PSD-95 expression in the hippocampus. (A) Representative gel pattern showing PSD-95 and $\beta$-actin protein bands after PEI, pcDNA3 plasmid, and SGKS422D transfection to the CA1 area. (B) Statistical analyses revealed that SGKS422D transfection significantly increased the PSD-95 level in the hippocampus ( ${ }^{* *} P<0.01$ when compared with the pcDNA3 group). pcDNA3 plasmid transfection alone did not have an effect on PSD-95 expression ( $P>0.05) . N=5$ each group. Data are mean \pm SEM.

$(0.5 \mu \mathrm{L} / \mathrm{min})$ to examine its effect on LTP. For PSD-95 Western blot assay, SGKS422D $(1.2 \mu \mathrm{g})$ was transfected to the CA1 area bilaterally. The injection needle was left in place for $5 \mathrm{~min}$ to limit diffusion of the injected DNA. The area of DNA transfection was examined by immunohistochemistry.

Brain sections were rinsed with $1 \times \mathrm{PBS}$ for $10 \mathrm{~min}$ at room temperature and permeabilized with pre-cold $\mathrm{EtOH} / \mathrm{CH}_{3} \mathrm{COOH}$ (95\%:5\%) for $10 \mathrm{~min}$, followed by $1 \times \mathrm{PBS}$ for $10 \mathrm{~min}$ three times. The sections were pre-incubated in a blocking solution containing 3\% normal goat serum, 3\% BSA, and $0.2 \%$ Triton $\mathrm{X}-100$ in $1 \times \mathrm{PBS}$ for $2 \mathrm{~h}$ at room temperature, followed by $1 \times \mathrm{PBS}$ for 10 min three times. For immunofluorescence analysis, tissue sections were incubated with a mouse anti-HA antibody (1:100; Roche Molecular Biochemicals) in blocking buffer at $4^{\circ} \mathrm{C}$ overnight. Sections were washed three times in $1 \times$ PBS and then incubated with goat anti-mouse FITC-conjugated IgG antibody (1:100; Sigma) in $1 \times$ PBS for $1 \mathrm{~h}$ at room temperature. Sections were washed three times in $1 \times \mathrm{PBS}$ and mounted with mounting medium.

The procedure used for LTP recording in CA1 neurons was according to that of Morgan and Teyler (1999). Rats were anesthetized with urethane $(1.4 \mathrm{mg} / \mathrm{kg}$, i.p.) and placed on a stereotaxic instrument. Platinum concentric bipolar-stimulating electrodes were positioned unilaterally (the DNA transfection side) to the Schaffer collateral/commisural pathway at $3.5 \mathrm{~mm}$ posterior to bregma, $3.0 \mathrm{~mm}$ lateral to the midline, and $2.6 \mathrm{~mm}$ below the skull surface. The recording electrodes were positioned $3.8 \mathrm{~mm}$ posterior to bregma and $2.5 \mathrm{~mm}$ from the midline. Electrodes were positioned to the stratum radiatum area of CA1. Test stimulation was $0.2 \mathrm{msec}$ in duration pulses delivered at a frequency of $0.033 \mathrm{~Hz}$. Stimulus intensities were adjusted to elicit a field excitatory postsynaptic potential (fEPSP), which corresponded to $\sim 70 \%$ of maximal fEPSP. LTP was induced with a tetanus of 200 $\mathrm{Hz} / 100 \mathrm{msec}$ every $2 \mathrm{sec}$, repeated 10 times, at a stimulation intensity the same as that of test stimulation. pcDNA3 vector or SGKS422A plasmid was transfected to the CA1 area $48 \mathrm{~h}$ before LTP recording.

For Western blotting, the hippocampal tissue was homog- enized in a lysis buffer containing $50 \mathrm{mM}$ Tris- $\mathrm{HCl}$ (pH 7.2), 150 $\mathrm{mM} \mathrm{NaCl}, 2 \mathrm{mM}$ EDTA, 1\% Igepal CA-630, $1 \mathrm{mM} \mathrm{Na}_{3} \mathrm{VO}_{4}, 50$ $\mathrm{mM} \mathrm{NaF}, 1 \mathrm{mM}$ PMSF, $20 \mu \mathrm{g} / \mathrm{mL}$ pepstatin A, $20 \mu \mathrm{g} / \mathrm{mL}$ leupeptin, and $20 \mu \mathrm{g} / \mathrm{mL}$ aprotinin. The lysate was centrifuged at $13,000 \mathrm{~g}$ for $10 \mathrm{~min}$ at $4^{\circ} \mathrm{C}$, and the supernatant was collected for protein concentration determination. Equal amounts of protein extract $(20 \mu \mathrm{g})$ were subject to 8\% SDS-polyacrylamide gel and transferred onto a PVDF membrane. The membrane was blocked with $2 \%$ BSA in $0.05 \%$ TBST for $1 \mathrm{~h}$ at room temperature and then immunoblotted with the following antibodies: phospho422SGK (1:500; Upstate); phospho-473Akt (1:1000; Cell Signaling); PSD-95 (1:2000; Upstate) at $4^{\circ} \mathrm{C}$ overnight. After washing with $0.1 \%$ TBST, the membrane was incubated with HRPconjugated secondary antibody for $1 \mathrm{~h}$ at room temperature and exposed to chemiluminescence substrate (ECL PLUS; Amersham). For re-probing to different antibodies-SGK antibody (1:1000; Upstate), Akt antibody (1:1000; Cell Signaling), and $\beta$-actin antibody (1:10,000; Chemicon)—the blot was stripped in $0.2 \mathrm{M}$ glycine ( $\mathrm{pH} 2.5$ ) and $0.05 \%$ Tween 20 at $80^{\circ} \mathrm{C}$ for $20 \mathrm{~min}$, then rinsed twice with $0.09 \mathrm{M}$ boric acid ( $\mathrm{pH} 7.4), 0.9 \% \mathrm{NaCl}$, and $0.05 \%$ Tween 20 . The protein bands were quantified by using the NIH Image J Software.

\section{Acknowledgments}

This work was supported by a Grant (NSC94-2321-B-001-009) from the National Science Council of Taiwan, Republic of China.

\section{References}

Abadall, B., Hassan, A., Benoist, C., Goula, D., Behr, J.P., and Demeneix B.A. 1996. A powerful nonviral vector for in vivo gene transfer into the adult mammalian brain: Polyethylenimine. Hum. Gene Ther. 7: 1947-1954.

Akama, K.T. and McEwen, B.S. 2003. Estrogen stimulates postsynaptic density-95 rapid protein synthesis via the Akt/protein kinase B pathway. J. Neurosci. 23: 2333-2339.

Beique, J.C. and Andrade, R. 2003. PSD-95 regulates synaptic transmission and plasticity in rat cerebral cortex. J. Physiol. 546: 859-867.

Bejar, R., Yasuda, R., Krugers, H., Hood, K., and Mayford, M. 2002. Transgenic calmodulin-dependent protein kinase II activation: Dose-dependent effects on synaptic plasticity, learning, and memory. J. Neurosci. 22: 5719-5726.

Bliss, T.V. and Collingridge, G.L. 1993. A synaptic model of memory: Long-term potentiation in the hippocampus. Nature 361: 31-39.

Bolshakov, V.Y., Golan, H., Kandel, E.R., and Siegelbaum, S.A. 1997. Recruitment of new sites of synaptic transmission during the cAMP-dependent late phase of LTP at CA3-CA1 synapses in the hippocampus. Neuron 19: 635-651.

Brazil, D.P. and Hemmings, B.A. 2001. Ten years of protein kinase B signaling: A hard Akt to follow. Trends Biochem. Sci. 26: 657-664.

Buchs, P.A. and Muller, D. 1996. Induction of long-term potentiation is associated with major ultrastructural changes of activated synapses. Proc. Natl. Acad. Sci. 93: 8040-8045.

David, S., Stegenga, S.L., Hu, P., Xiong, G., Kerr, E., Becker, K.B., Venkatapathy, S., Warrington, J.A., and Kalb, R.G. 2005. Expression of serum- and glucocorticoid-inducible kinase is regulated in an experience-dependent manner and can cause dendrite growth. $J$. Neurosci. 25: 7048-7053.

Ehrlich, I. and Malinow, R. 2004. Postsynaptic density 95 controls AMPA receptor incorporation during LTP and experience-driven synaptic plasticity. J. Neurosci. 24: 916-927.

English, J.D. and Sweatt, J.D. 1997. A requirement for the mitogen-activated protein kinase cascade in hippocampal long-term potentiation. J. Biol. Chem. 272: 19103-19106.

Geinisman, Y., Detoledo-Morrell, L., Morrell, F., Persina, I.S., and Beatty, M.A. 1996. Synapse restructuring associated with the maintenance phase of hippocampal long-term potentiation. J. Comp. Neurol. 368: 413-423.

Kobayashi, T. and Cohen, P. 1999. Activation of serum- and glucocorticoid-regulated protein kinase by agonists that activate phosphatidylinositide 3-kinase is mediated by

3-phosphoinositide-dependent protein kinase-1 (PDK1) and PDK2. Biochem. I. 339: 319-328.

Lee, E.H.Y., Hsu, W.L., Ma, Y.L., Lee, P.J., and Chao, C.C. 2003. 
Enrichment enhances the expression of $s g k$, a glucocorticoid-induced gene, and facilitates spatial learning through glutamate AMPA receptor mediation. Eur. J. Neurosci. 18: 2842-2852.

Lee, C.C., Huang, C.C., Wu, M.Y., and Hsu, K.S. 2005. Insulin stimulates postsynaptic density-95 protein translation via the phosphoinositide 3-kinase-Akt-mammalian target of rapamycin signaling pathway. J. Biol. Chem. 280: 18543-18550.

Lin, C.H., Yeh, S.H., Lin, C.H., Lu, K.T., Leu, T.H., Chang, W.C., and Gean, P.W. 2001. A role for the PI-3 kinase signaling pathway in fear conditioning and synaptic plasticity in the amygdala. Neuron 31: $841-851$.

Mizuno, H. and Nishida, E. 2001. The ERK MAP kinase pathway mediates induction of SGK (serum- and glucocorticoid-inducible kinase) by growth factors. Genes Cells 6: 261-268.

Morgan, S.L. and Teyler, T.J. 1999. VDCCs and NMDARs underlie two forms of LTP in CA1 hippocampus in vivo. J. Neurophysiol. 82: 736-740.

Naray-Fejes-Toth, A., Fejes-Toth, G., Volk, K.A., and Stokes, J.B. 2000. SGK is a primary glucocorticoid-induced gene in the human. $J$. Steroid Biochem. Mol. Biol. 75: 51-56.

Nayak, A., Zastrow, D.J., Lickteig, R., Zahniser, N.R., and Browning, M.D. 1998. Maintenance of late-phase LTP is accompanied by PKA-dependent increase in AMPA receptor synthesis. Nature 394: $680-683$.

Nguyen, P.V. and Woo, N.H. 2003. Regulation of hippocampal synaptic plasticity by cyclic AMP-dependent protein kinases. Prog. Neurobiol. 71: $401-437$.

Okabe, S., Kim, H.D., Miwa, A., Kuriu, T., and Okado, H. 1999. Continual remodeling of postsynaptic density and its regulation by synaptic activity. Nat. Neurosci. 2: 804-811.

Opazo, P., Watabe, A.M., Grant, S.G., and O'Dell, T.J. 2003.

Phosphatidylinositol 3-kinase regulates the induction of long-term potentiation through extracellular signal-related kinase-independent mechanisms. J. Neurosci. 23: 3679-3688.

Park, J., Leong, M.L., Buse, P., Maiyar, A.C., Firestone, G.L., and Hemmings, B.A. 1999. Serum and glucocorticoid-inducible kinase (SGK) is a target of the PI3-kinase-stimulating signaling pathway. EMBO J. 18: 3024-3033.

Pearce, D., Verrey, F., Chen, S.Y., Mastroberardino, L., Meijer, O.C., Wang, J., and Bhargava, A. 2000. Role of SGK in mineralocorticoid-regulated sodium transport. Kidney Int. 57: 1283-1289.

Roozendaal, B. 2000. Glucocorticoid and the regulation of memory consolidation. Psychoneuroendocrinology 25: 213-238.

Sanna, P.P., Cammalleri, M., Berton, F., Simpson, C., Lutjens, R., Bloom, F.E., and Francesconi, W. 2002. Phosphatidylinositol 3-kinase is required for the expression but not for the induction or the maintenance of long-term potentiation in the hippocampal CA1 region. J. Neurosci. 22: 3359-3365.

Tsai, K.J., Chen, S.K., Ma, Y.L., Hsu, W.L., and Lee, E.H.Y. 2002. $s g k$, a primary glucocorticoid-induced gene, facilitates memory consolidation of spatial learning in rats. Proc. Natl. Acad. Sci. 99: 3990-3995.

Waldegger, S., Barth, P., Raber, G., and Lang, F. 1997. Cloning and characterization of a putative human serine/threonine protein kinase transcriptionally modified during anisotonic and isotonic alterations of cell volume. Proc. Natl. Acad. Sci. 94: 4440-4445.

Webster, M.K., Goya, L., Ge, Y., Maiyar, A.C., and Firestone, G.L. 1993. Characterization of $s g k$, a novel member of the serine/threonine protein kinase gene family which is transcriptionally induced by glucocorticoids and serum. Mol. Cell. Biol. 13: 2031-2040.

Received October 5, 2005; accepted in revised form January 17, 2006.
118 Learning \& Memory www.learnmem.org 


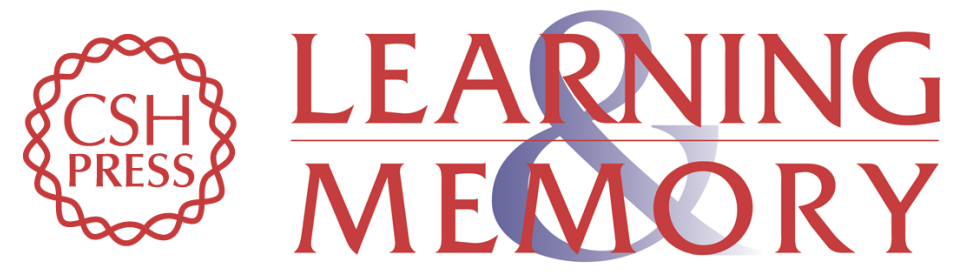

\section{SGK protein kinase facilitates the expression of long-term potentiation in hippocampal neurons}

Yun L. Ma, Ming C. Tsai, Wei L. Hsu, et al.

Learn. Mem. 2006, 13:

Access the most recent version at doi:10.1101//m.179206

References This article cites 30 articles, 14 of which can be accessed free at:

http://learnmem.cshlp.org/content/13/2/114.full.html\#ref-list-1

License

Email Alerting Receive free email alerts when new articles cite this article - sign up in the box at the Service top right corner of the article or click here. 